\title{
pORF5 plasmid protein of Chlamydia trachomatis induces MAPK-mediated pro-inflammatory cytokines via TLR2 activation in THP-1 cells
}

\author{
ZHOU Hui ${ }^{1,2 \dagger}$, HUANG QiuLin ${ }^{3 \dagger}$, LI ZhongYu ${ }^{1 *}$, WU YiMou ${ }^{1}$, XIE XiaoBing ${ }^{2}$, \\ MA KangKang ${ }^{1}$, CAO WenJuan ${ }^{1}$, ZHOU Zhou $^{1}$, LU ChunXue $^{1} \&$ ZHONG GuangMing ${ }^{4}$ \\ ${ }^{1}$ Pathogenic Biology Institute, University of South China, Hengyang 421001, China; \\ ${ }^{2}$ Department of Laboratory Medicine, the First Hospital of Hunan University of Chinese Medicine, Changsha 410007, China; \\ ${ }^{3}$ Department of General Surgery, the First Affiliated Hospital of University of South China, Hengyang 421001, China; \\ ${ }^{4}$ Department of Microbiology and Immunology, University of Texas Health Science Center, San Antonio, TX 78229, USA
}

Received February 27, 2013; accepted March 13, 2013; published online March 27, 2013

\begin{abstract}
Infection with Chlamydia trachomatis induces inflammatory pathologies in the urogenital tract that can lead to infertility and ectopic pregnancy. Pathogenesis of infection has been mostly attributed to excessive cytokine production. However, precise mechanisms on how $C$. trachomatis triggers this production, and which protein(s) stimulate inflammatory cytokines remains unknown. In the present study, the $C$. trachomatis pORF5 protein induced tumor necrosis factor alpha (TNF- $\alpha$ ), interleukin-1 beta (IL-1 $\beta$ ) and interleukin-8 (IL-8) in dose- and time-dependent manners in the THP-1 human monocyte cell line. We found that intracellular p38/mitogen-activated protein kinase (MAPK) and extracellular signal-regulated kinase (ERK)/MAPK signaling pathways were required for the induction of TNF- $\alpha$, IL-1 $\beta$ and IL-8. Blockade of toll-like receptor 2 (TLR2) signaling reduced induction levels of TNF- $\alpha$, IL- 8 and IL-1 $\beta$. We concluded that the $C$. trachomatis pORF5 protein might contribute to the inflammatory processes associated with chlamydial infections.
\end{abstract}

Chlamydia trachomatis, pORF5 plasmid protein, mitogen-activated protein kinase, proinflammatory cytokines, TLR2

Citation: Zhou H, Huang Q L, Li Z Y, et al. pORF5 plasmid protein of Chlamydia trachomatis induces MAPK-mediated pro-inflammatory cytokines via TLR2 activation in THP-1 cells. Sci China Life Sci, 2013, 56: 460-466, doi: 10.1007/s11427-013-4470-8

Chlamydia trachomatis is a pathogenic microorganism responsible for ocular and sexually transmitted infections. Trachoma caused by serovars A, B, Ba and C, is the world's leading cause of infectious blindness [1]. Infections with genital serovars D-K mostly manifest as urethritis, prostatitis or epididymitis in men, and as cervicitis, salpingitis or endometritis in women [2,3]. The L serovars, including L1, L2, L2a and L3, often cause disseminated diseases. Chlamydial genital tract infection may also significantly increase

$\dagger$ Contributed equally to this work

*Corresponding author (email: lzhy1023@hotmail.com) the risk for HIV infection and cervical carcinoma [4-6]. The majority of chlamydial infections are asymptomatic, therefore infected individuals often do not receive timely medical treatment, and develop a chronic persistent infection leading to severe complications. Therefore, research on chlamydial virulence factors and pathogenic mechanisms is required to improve diagnosis and prevention of chlamydial infections.

Infection with $C$. trachomatis induces a wide array of inflammatory cytokines and chemokines [7-9]. These likely contribute to chlamydia-induced inflammatory pathologies [10]. The pathologies associated with genital infection and 
ocular trachoma are thought to result from injury during an aggressive inflammatory response. However, the precise mechanisms related to how chlamydia triggers such a response, and which protein(s) stimulate inflammatory cytokines remains unclear.

Along with a highly conserved genome of around $1000 \mathrm{~kb}$ of chromosomal DNA, all $C$. trachomatis serovars also contain a $7.5 \mathrm{~kb}$ cryptic plasmid [11]. This plasmid encodes eight open reading frames, designated pORF1-8. Although the function of the plasmid has not been mapped, evidence has shown that plasmid-deficient chlamydia fail to induce any immune pathology [12]. This would suggest that the plasmid might be associated with virulence. As the only secreted protein encoded by the plasmid, pORF5 possibly contributes to chlamydia-induced pathologies.

The aims of our study were to determine whether pORF5 can activate monocytes to produce pro-inflammatory cytokines (TNF- $\alpha$, IL-1 $\beta$ and IL- 8 ); and determine whether TLR2 and MAPKs are required for pORF5-induced cytokine expression by monocyte cells. A recombinant pORF5 protein was produced and purified, and then human monocyte cells were incubated with pORF5 in the presence or absence of anti-TLR antibodies and specific inhibitors of MAPKs. We evaluated the effects of pORF5 on IL-1 $\beta$, IL-8 and TNF- $\alpha$ production.

\section{Materials and methods}

\subsection{Expression and purification of GST-pORF5 fusion protein}

A pGEX-6p/pORF5 recombinant plasmid was transformed into Escherichia coli XL1-Blue host cells for expression of the glutathione $S$ transferase (GST)-pORF5 fusion protein. Expression was induced with isopropyl- $\beta$-D-thiogalactopyranoside (IPTG; Invitrogen, Carlsbad, CA), and the fusion protein extracted by lysing $E$. coli in a lysis buffer (1\% Triton X-100, $1 \mathrm{mmol} \mathrm{L}^{-1}$ phenylmethylsulfonyl fluoride, $75 \mathrm{U} \mathrm{mL}^{-1}$ of aprotinin, $20 \mu \mathrm{mol} \mathrm{\textrm {L } ^ { - 1 }}$ leupeptin, and 1.6 $\mu \mathrm{mol} \mathrm{L}^{-1}$ pepstatin) and subjecting to sonication. After high-speed centrifugation $(12000 \times g)$ to remove debris, the fusion protein was purified with glutathione-conjugated agarose beads (Amersham Pharmacia Biotech, Inc., Piscataway, NJ), and subjected to cleavage of the GST tag with PreScission protease (Pharmacia) following the manufacturer's instructions. After concentrating with a Centricon YM-10 (Millipore, Billerica, MA) and removing endotoxins with polymyxin B (Sigma, St. Louis, MO), the purified pORF5 protein was ready to use in macrophage stimulation experiments.

\subsection{Cell culture and simulation}

THP-1 cells (TIB-202; ATCC) were cultured in RPMI 1640 (GIBCO BRL, Rockville, MD) supplemented with $10 \%$ fetal bovine serum (FBS; GIBCO BRL) at $37^{\circ} \mathrm{C} / 5 \% \quad \mathrm{CO}_{2}$. For stimulation experiments, THP-1 cells were seeded in 24-well plates at a density of $10^{5}$ cells $\mathrm{mL}^{-1}$ and cultivated in $1 \%$ FBS overnight. The purified pORF5 was added to cultures as stimuli at the concentrations and times indicated for individual experiments. The E. coli lipopolysaccharide (LPS; Sigma) was used as a positive control stimulus. In some experiments, cells were pretreated with mouse a antiTLR2 antibody (Abcam, Cambridge, UK), PD98059 (Sigma) and SB203580 (Sigma) for $30 \mathrm{~min}$ prior to pORF5 stimulation.

\subsection{ELISA for cytokine detection}

At the end of culture, the cells were lysed by two consecutive cycles of freezing/thawing to yield maximal recovery of total cytokines. This included both intracellular cytokines and those released into the cell culture. The concentrations of TNF- $\alpha$, IL- $1 \beta$ and IL- 8 were measured using human ELISA kits (R\&D Systems, Inc., Minneapolis, MN), according to the manufacturer's instructions. Briefly, 96-well ELISA microplates were coated with a capture antibody, and after blocking with 5\% BSA, cytokine samples were added to the coated plates. This was followed with a biotinconjugated detection antibody. Antibody binding was measured with horseradish peroxidase-conjugated avidin along with a soluble colorimetric substrate. The absorbance was measured at $405 \mathrm{~nm}$ using a microplate reader (Molecular Devices Corporation, Sunnyvale, CA) and cytokine concentrations calculated.

\subsection{SDS-PAGE and Western blotting}

Cell samples were harvested and solubilized with $2 \%$ sodium dodecyl sulfate (SDS) sample buffer, then loaded onto $12 \%$ SDS polyacrylamide gels. After electrophoresis, proteins were transferred onto nitrocellulose membranes, and incubated with primary antibodies, including rabbit monoclonal antibodies against phospho-ERK, phospho-JNK and phospho-p38 (Cell Signaling Technology, Beverly, MA), and mouse monoclonal antibodies against JNK, p38, and ERK (Santa Cruz Biotechnology Inc., Santa Cruz, CA). Following incubation with the primary antibody, blots were incubated with a secondary antibody conjugated to horseradish peroxidase (either goat anti-rabbit or goat anti-mouse immunoglobulin G; Jackson Immunologicals, Westgrove, PA) and visualized using an enhanced chemiluminescence (ECL) kit (Santa Cruz Biotech).

\subsection{Statistical analysis}

Data from three independent experiments are presented as the mean \pm standard deviation. Statistical software (SPSS13.0) was used to analyze results with Student's $t$-test followed by one-way analysis of variance (ANOVA). A $P$ - 
value less than 0.05 was considered statistically significant.

\section{Results}

\subsection{Expression and purification of pORF5 protein}

The GST-pORF5 fusion protein was expressed in a prokaryotic expression system and purified with glutathione agarose beads. The purified GST-pORF5 was cleaved with PreScission protease to remove the GST tag. We observed the tagged and purified pORF5 (54 kD), along with the cleaved pORF5 (28 kD) on SDS-PAGE gels (Figure 1A). This result was confirmed by Western blotting analysis using the specific monoclonal mouse anti-pORF5 antibody (clone 2H4) (Figure 1B).

\section{2 pORF5 protein induces inflammatory cytokines in THP-1 cells}

The purified pORF5 induced TNF- $\alpha$, IL- $1 \beta$ and IL- 8 production in THP- 1 cells in a dose-dependent manner, for the concentration range 1-8 $\mu \mathrm{g} \mathrm{mL}^{-1}$ (Figure 2A). From 1-6 $\mu \mathrm{g} \mathrm{mL} \mathrm{m}^{-1}$, a correlation between dose and cytokine production was observed. Low concentrations of pORF5 induced low levels of cytokine secretion, while higher concentrations induced higher levels of cytokine production. When the concentration was greater than $6 \mu \mathrm{g} \mathrm{mL}^{-1}$, production of TNF- $\alpha$, IL- $1 \beta$ and IL- 8 decreased. Cytokine production was also time-dependent from $6-36 \mathrm{~h}$, with TNF- $\alpha$ levels peaking at $6 \mathrm{~h}$ post-stimulation. The peak amounts of IL- $1 \beta$ and IL-8 occurred at $12 \mathrm{~h}$ post-stimulation (Figure $2 \mathrm{~B}$ ). In all experiments, E. coli LPS was used as the positive control, as this is the most effective stimuli in triggering cytokine production. The negative control, phosphate-buffered saline (PBS), barely induced any expression of cytokines.

No endotoxins were detected using a Limulus amebocyte lysate assay for the purified pORF5 protein. The possibility that its pro-inflammatory activity was attributed to LPS contamination was further investigated by testing pORF5 sensitivity to polymyxin B. As shown in Figure 2C, pORF5 pre-treated with $50 \mu \mathrm{g} \mathrm{mL}^{-1}$ polymyxin B had no effect on TNF- $\alpha$ production $(P>0.05)$ while blocking most of the LPS-mediated stimulation activity $(P<0.01)$.

\section{3 pORF5 activates ERK and p38/MAPK signaling pathways}

As shown in Figure 3, pORF5 activated p38/MAPK and ERK/MAPK pathways, but was unable to activate the JNK/MAPK. The p38/MAPK and ERK/MAPK pathways were activated at about 15 min after pORF5 stimulation, peaking at 15 and $30 \mathrm{~min}$, respectively, then gradually decreased.

\section{4 p38/MAPK and ERK/MAPK pathways are in- volved in pro-inflammatory cytokine production in- duced by pORF5 protein}

After pretreating with SB202190, a p38/MAPK inhibitor, and PD98059, an ERK/MAPK inhibitor, at three different doses $\left(1,10\right.$ and $\left.30 \mu \mathrm{mol} \mathrm{L}^{-1}\right)$ for $30 \mathrm{~min}$, THP-1 cells were stimulated with $6 \mu \mathrm{g} \mathrm{mL}{ }^{-1}$ pORF5 for $12 \mathrm{~h}$. We then examined the levels of TNF- $\alpha$, IL- $1 \beta$ and IL- 8 to assess the effects on the production of different cytokines. As shown in Figure 4, inhibition of p38/MAPK and ERK/MAPK pathways reduced pORF5-induced TNF- $\alpha$, IL- $1 \beta$ and IL- 8 production levels. SB202190 and PD98059 effected proinflammatory cytokine production in a dose-dependent manner. Compared with the control group, the levels of TNF- $\alpha$, IL- $1 \beta$ and IL- 8 were reduced to $22.4 \%, 27.6 \%$ and $22.8 \%$ respectively, when $30 \mu \mathrm{mol} \mathrm{L}^{-1} \mathrm{SB} 202190$ was used (Figure 4A). When $30 \mu \mathrm{mol} \mathrm{L}^{-1}$ PD98059 was used, TNF- $\alpha$, IL- $1 \beta$ and IL- 8 levels were reduced by $60.3 \%, 58.8 \%$ and $45.7 \%$ (Figure 4B).
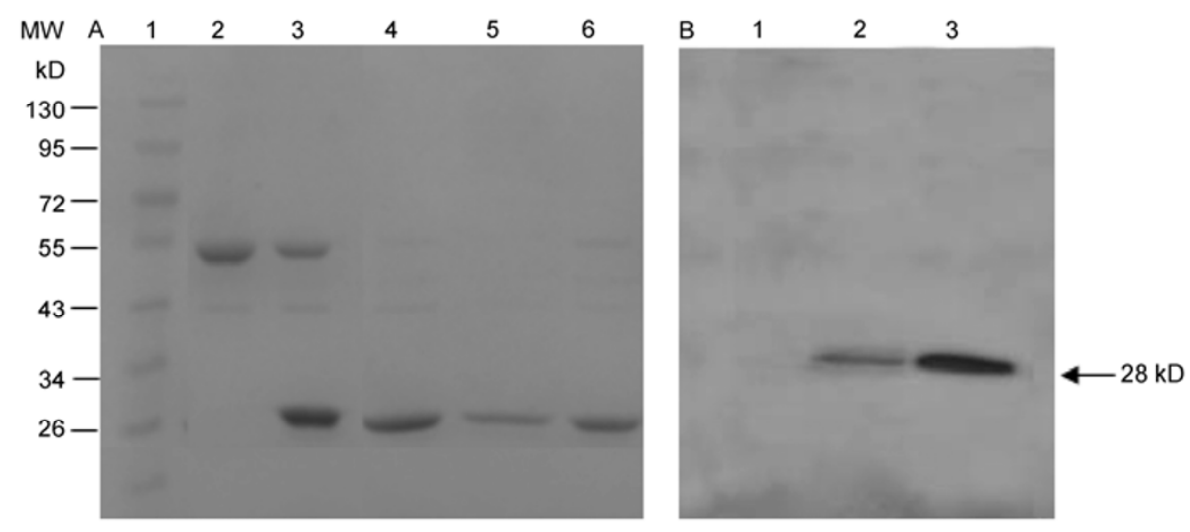

Figure 1 Purification and identification of the pORF5 protein. A, Coomassie blue-stained acrylamide gel after SDS-PAGE. Lane 1, marker; 2, GST-pORF5; 3, incompletely cleaved GST-pORF5; 4, pORF5 eluate 1; 5, pORF5 eluate 2; 6, pORF5 eluate 3. B, Western blotting assay. Lane 1, XL1-Blue E. coli lysate; 2, eluted GST-pORF5 agarose beads after protease cleavage; 3, supernatant of GST-pORF5 agarose beads after cleavage with protease. 

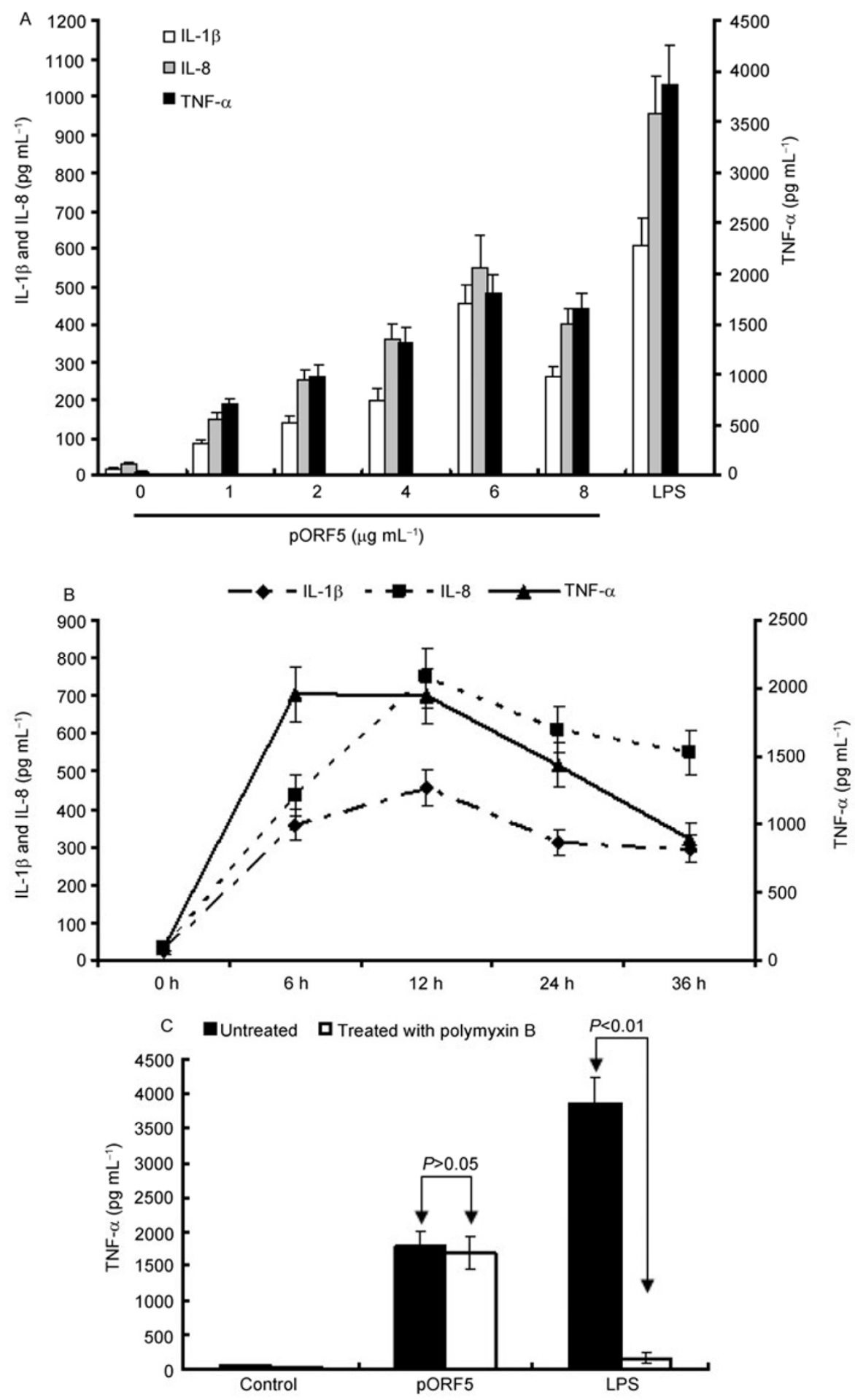

Figure 2 Induction of inflammatory cytokines by the pORF5 protein. A, Dose-dependent effects of purified pORF5 on the production of TNF- $\alpha$, IL-1 $\beta$ and IL-8 in THP-1 cells. THP-1 cells were incubated for $12 \mathrm{~h}$ with or without pORF5 at final concentrations ranging from $2-8 \mu \mathrm{g} \mathrm{mL}{ }^{-1}$. LPS at a final concentration of $100 \mathrm{ng} \mathrm{mL}^{-1}$ was used as a positive control, and PBS was used as a negative control. Samples were collected and assayed for TNF- $\alpha$, IL- $1 \beta$ and IL-8 production. Data were obtained from three independent experiments. The error bars indicated standard deviations. B, Time-dependent effects of purified pORF5 on TNF- $\alpha$, IL-1 $\beta$ and IL-8 production in THP-1 cells. THP- 1 cells were incubated with $6 \mu \mathrm{gL} \mathrm{mL}^{-1}$ pORF5 for $0-36 \mathrm{~h}$. Samples were harvested and assayed for TNF- $\alpha$, IL-1 $\beta$ and IL-8 production. Data are expressed as mean \pm SD $(n=3)$. Error bars indicate standard deviations. C, Effects of polymyxin B on pORF5- and LPS-induced TNF- $\alpha$ production in THP-1 cells. pORF5 or LPS was pre-treated with $50 \mu \mathrm{gL}^{-1}$ polymyxin B for $1 \mathrm{~h}$ before stimulating THP-1 cells. pORF5 $\left(6 \mu \mathrm{g} \mathrm{mL}^{-1}\right)$ or LPS $\left(100 \mathrm{ng} \mathrm{mL}^{-1}\right)$ were then added to culture medium. After a 12-h incubation, samples were harvested and the concentration of TNF- $\alpha$ determined by ELISA. The data were from three independent experiments. Error bars indicate standard deviations. 


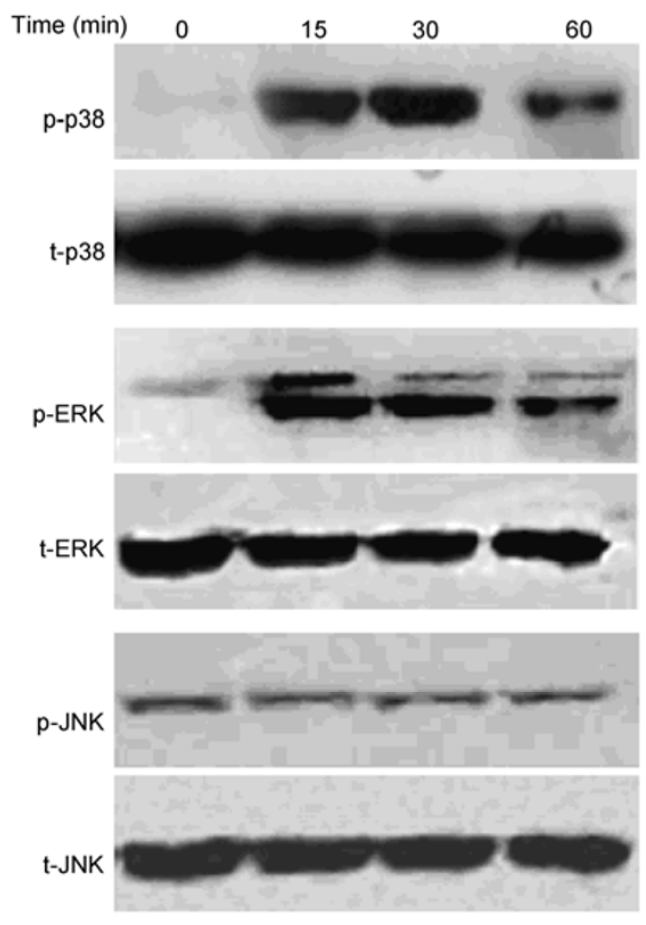

Figure 3 Activation of MAPKs induced by pORF5 in THP-1 cells. THP-1 cells were incubated with $6 \mu \mathrm{g} \mathrm{mL} \mathrm{m}^{-1}$ pORF5 for 0-60 min. Activation of p38, ERK and JNK was detected by Western blotting using the appropriate specific antibodies.
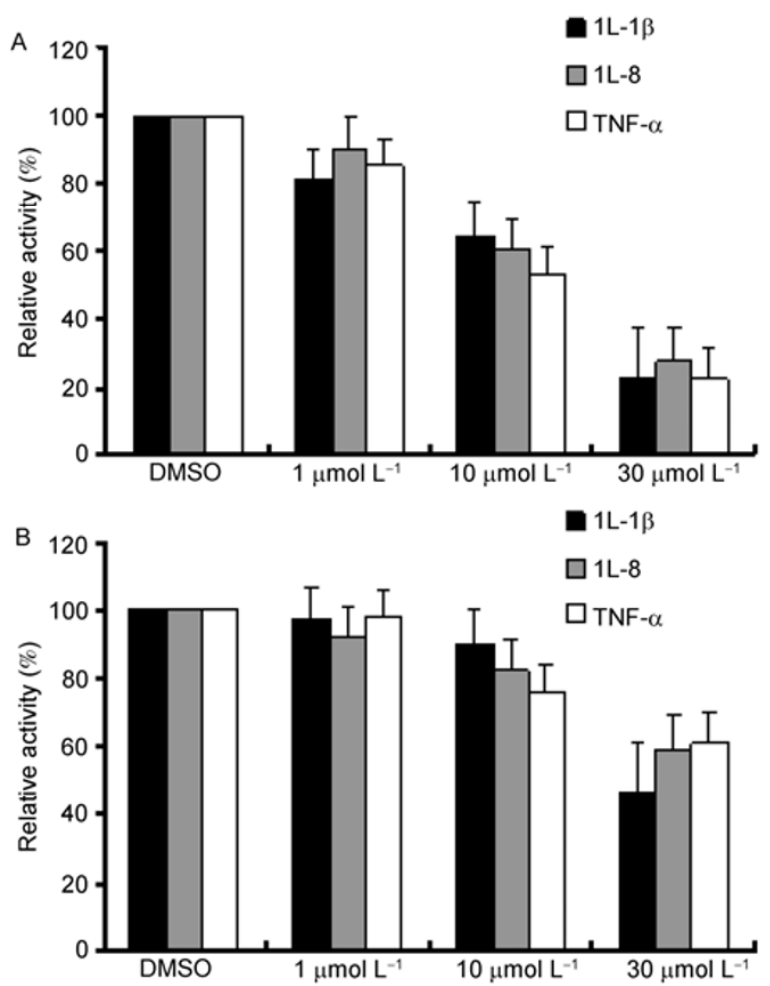

Figure 4 Effects of specific MAPK inhibitors on pORF5-induced cytokine production. THP-1 cells were pre-incubated with three different concentrations of SB202190 (A), PD98059 (B) or dimethyl sulfoxide (DMSO) for $30 \mathrm{~min}$. We then added $6 \mu \mathrm{g} \mathrm{mL}^{-1}$ pORF5 to the medium. TNF- $\alpha$, IL-1 $\beta$ and IL- 8 levels were detected by ELISA. Our results were representative of three independent experiments.

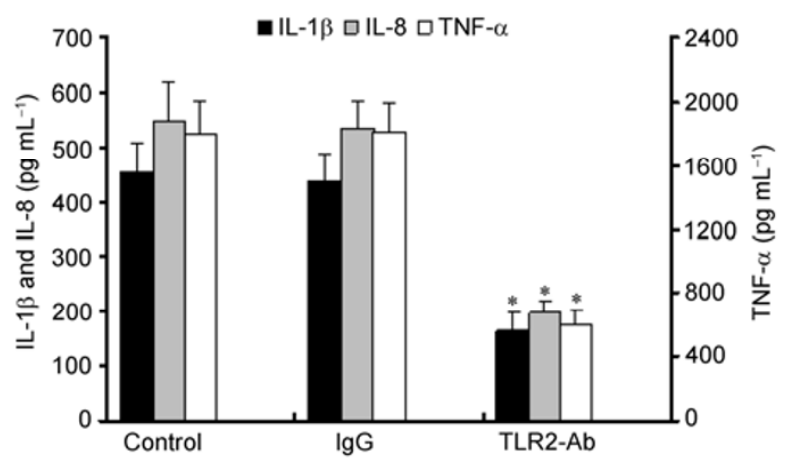

Figure 5 TLR2 is involved in the production of TNF- $\alpha$, IL-1 $\beta$ and IL-8. THP-1 cells were pre-incubated with $10 \mu \mathrm{g} \mathrm{mL}{ }^{-1}$ anti-TLR2, or isotypematched mouse IgG for $30 \mathrm{~min}$. Purified pORF5 was added at a final concentration of $6.0 \mu \mathrm{g} \mathrm{mL} \mathrm{m}^{-1}$. TNF- $\alpha$, IL-1 $\beta$ and IL-8 concentrations were determined by ELISA. *, $P<0.01$ compared with the control groups.

\subsection{TLR2 is involved in pORF5-induced proinflam- matory cytokine production}

Compared with the control group, blocking TLR2 with a neutralizing anti-human TLR2 antibody resulted in significant reduction of pro-inflammatory cytokine production induced by pORF5 $(P<0.01)$. The TLR2 antibody had negligible effect on LPS-inducing cytokine production (data not shown). The control IgG did not influence the production of IL-1 $\beta$, IL-8 and TNF- $\alpha$ (Figure 5).

\section{Discussion}

C. trachomatis is an obligate intracellular bacterial pathogen. During intravacuolar growth, $C$. trachomatis must secrete factors into the inclusion membrane [13-15] or the host cell cytosol [16-18] for interacting with and/or manipulating host cell signaling pathways [19-21]. As a result of chlamydia-host interactions, host cell genes encoding inflammatory cytokines and chemokines are often activated. Previous studies have been shown that a wide array of inflammatory cytokines and chemokines, including IL- $1 \alpha$, IL- 6 , IL-8, IL-18, TNF- $\alpha$ [7-9], are produced in vitro and/or in vivo. These are believed to contribute to chlamydia-induced pathologies [10].

There are 19 different serovars among C. trachomatis species. Almost all serovars contain the $7.5 \mathrm{~kb}$ cryptic plasmid [11]. Loss of this plasmid has been associated with immunopathology and infectivity [22,23]. Vaginal inoculation of mice with a plasmid-cured derivative of $C$. muridarum reduced bacterial burden almost 10 -fold, and there was an associated absence of oviduct pathology [12]. Other research has also indicated that plasmid-negative strains had significantly higher $50 \%$ infective dose values relative to a wild-type matched isolate [24]. These results indicate that this cryptic plasmid might be associated with virulence attributes. Of the eight plasmid-encoded proteins, pORF5 has 
been shown to be the only protein that is secreted and distributed in the cytosol of infected cells. The remaining seven proteins localize inside chlamydial inclusions [17]. Since Chlamydia restricts its own biosynthetic activity within an inclusion, pORF5 might be relevant to chlamydial interactions with host cells. Our findings show that purified pORF5 induced TNF- $\alpha$, IL- $1 \beta$ and IL- 8 production in doseand time-dependent manners. The inflammatory reactions peaked when the pORF5 concentration was $6 \mu \mathrm{g} \mathrm{mL}$. Higher concentrations of pORF5 were inhibitory to cytokine production, which may indicate some interference with the biological function of the cells. TNF- $\alpha$ peaked at $6 \mathrm{~h}$ post-stimulation, while IL- $1 \beta$ and IL- 8 production occurred $12 \mathrm{~h}$ after stimulation. Our observations clearly show pORF5 induces cytokine production, and suggest that pORF5 might contribute to chlamydia-induced inflammatory pathologies.

MAPKs comprise a family of proline-directed serine/ threonine kinases. These are important signaling pathways for the host immune response. Three major MAPKs, ERK, p38 and JNK have been identified in the intracellular network that transduces extracellular signals to an intracellular response. Previous studies have demonstrated that MAPKs contribute to the production of different cytokines during chlamydial infection $[25,26]$. We hypothesized that MAPKs were also involved in the production of TNF- $\alpha$, IL- $1 \beta$ and IL-8 induced by pORF5. This hypothesis was verified by our results derived from the phosphorylation detection and specific inhibitor studies. The effects of specific inhibitors on cytokine production varied. When p38/MAPK was blocked $30 \mu \mathrm{mol} \mathrm{L}{ }^{-1}$ SB202190, levels of TNF- $\alpha$, IL-1 $\beta$ and IL-8 were reduced. Blocking ERK/MAPK with $30 \mu \mathrm{mol} \mathrm{L}{ }^{-1}$ PD98059 further reduced TNF- $\alpha$, IL- $1 \beta$ and IL-8 levels. The p38/MAPK pathway appeared to be more sensitive to cytokine responses following pORF5 stimulation.

TLRs are transmembrane receptors within the IL- 1 receptor superfamily. They are regarded as the major molecules by which hosts recognize invading microorganisms. Recent experiments have shown that TLR2 plays a fundamental role in chlamydia pathology. TLR2-deficient mice did not develop oviduct pathology after infection, demonstrating that TLR2 signaling is directly involved in disease development [27,28]. Data from mouse models have demonstrated that plasmid-deficient $C$. muridarum failed to activate TLR2-dependent immune responses in vivo or in vitro [29]. These observations suggest that the plasmid regulates TLR2-dependent signaling and participates in chlamydial pathogenesis. We found that an anti-TLR2 antibody significantly inhibited inflammatory cytokine production. This indicates that TLR2 likely participates in the production of inflammatory cytokines induced by pORF5.

In summary, we have demonstrated that pORF5 induces expression of the pro-inflammatory cytokines TNF- $\alpha$, IL- $1 \beta$ and IL-8 in THP-1 cells. The induction of these cytokines has subsequent effects upon TLR2, p38/MAPK and ERK/
MAPK. However, further research upstream and downstream of the MAPK pathways, and the transcription factors need to be conducted. Our findings presented herein now provide a better understanding of cytokine release and the pathogenicity of $C$. trachomatis at a molecular level.

This work was supported by the National Natural Science Foundation of China (30970165, 81102230) and Team Project for the Technology Innovation of Higher Education of Hunan Province, China, 2010.

1 Mabey D C, Solomon A W, Foster A. Trachoma. Lancet, 2003, 362: 223-229

2 Brunham R C, Rey-Ladino J. Immunology of Chlamydia infection: implications for a Chlamydia trachomatis vaccine. Nat Rev Immunol, 2005, 5: 149-161

3 Belland R, Ojcius D M, Byrne G I. Chlamydia. Nat Rev Microbiol, 2004, 2: 530-531

4 Cristina Ferreira Silva L, Espinosa Miranda A, Santos Batalha R, et al. Chlamydia trachomatis infection among HIV-infected women attending an AIDS clinic in the city of Manaus, Brazil Braz J Infect Dis, 2012, 16: 335-338

5 Verteramo R, Pierangeli A, Mancini E, et al. Human papillomaviruses and genital co-infections in gynaecological outpatients. BMC Infect Dis, 2009, 9: 16-27

6 Oh J K, Franceschi S, Kim B K, et al. Prevalence of human papillomavirus and Chlamydia trachomatis infection among women attending cervical cancer screening in the Republic of Korea. Eur J Cancer Prev, 2009, 18: 56-61

7 Buchholz K R, Stephens R S. Activation of the host cell proinflammatory interleukin- 8 response by Chlamydia trachomatis. Cell Microbiol, 2006, 8: 1768-1779

8 Maxion H K, Kelly K A. Chemokine expression patterns differ within anatomically distinct regions of the genital tract during Chlamydia trachomatis infection. Infect Immun, 2002, 70: 1538-1546

9 Gervassi A, Alderson M R, Suchland R, et al. Differential regulation of inflammatory cytokine secretion by human dendritic cells upon Chlamydia trachomatis infection. Infect Immun, 2004, 72: 72317239

10 Stephens R S. The cellular paradigm of chlamydial pathogenesis. Trends Microbiol, 2003, 11: 44-51

11 Thomas N S, Lusher M, Storey C C, et al. Plasmid diversity in Chlamydia. Microbiology, 1997, 143: 1847-1854

12 O'Connell C M, Ingalls R R, Andrews C W Jr, et al. Plasmiddeficient Chlamydia muridarum fail to induce immune pathology and protect against oviduct disease. J Immunol, 2007, 179: 4027-4034

13 Li Z, Huang Q, Su S, et al. Localization and characterization of the hypothetical protein CT440 in Chlamydia trachomatis-infected cells. Sci China Life Sci, 2011, 54: 1048-1054

14 Li Z, Chen C, Chen D, et al. Characterization of fifty putative inclusion membrane proteins encoded in the Chlamydia trachomatis genome. Infect Immun, 2008, 76: 2746-2757

15 Chen C, Chen D, Sharma J, et al. The hypothetical protein CT813 is localized in the Chlamydia trachomatis inclusion membrane and is immunogenic in women urogenitally infected with $C$. trachomatis. Infect Immun, 2006, 74: 4826-4840

16 Zhong G. Chlamydia trachomatis secretion of proteases for manipulating host signaling pathways. Front Microbiol, 2011, 2: 14

17 Li Z, Chen D, Zhong Y, et al. The chlamydial plasmid-encoded protein pgp3 is secreted into the cytosol of Chlamydia-infected cells. Infect Immun, 2008, 76: 3415-3428

18 Lei L, Qi M, Budrys N, et al. Localization of Chlamydia trachomatis hypothetical protein CT311 in host cell cytoplasm. Microb Pathog, 2011, 51: 101-109

19 Greene W, Xiao Y, Huang Y, et al. Chlamydia-infected cells continue to undergo mitosis and resist induction of apoptosis. Infect Immun, 
2004, 72: 451-460

$20 \mathrm{Su} \mathrm{H}$, McClarty G, Dong F, et al. Activation of Raf/MEK/ERK/ cPLA2 signaling pathway is essential for chlamydial acquisition of host glycerophospholipids. J Biol Chem, 2004, 279: 9409-9416

21 Xiao Y, Zhong Y, Greene W, et al. Chlamydia trachomatis infection inhibits both Bax and Bak activation induced by staurosporine. Infect Immun, 2004, 72: 5470-5474

22 Russell M, Darville T, Chandra-Kuntal K, et al. Infectivity acts as in vivo selection for maintenance of the chlamydial cryptic plasmid. Infect Immun, 2011, 79: 98-107

23 O'Connell C M, Nicks K M. A plasmid-cured Chlamydia muridarum strain displays altered plaque morphology and reduced infectivity in cell culture. Microbiology, 2006, 152: 1601-1607

24 Carlson J H, Whitmire W M, Crane D D, et al. The Chlamydia trachomatis plasmid is a transcriptional regulator of chromosomal genes and a virulence factor. Infect Immun, 2008, 76: 2273-2283

25 Srivastava P, Vardhan H, Bhengraj A R, et al. Azithromycin treatment modulates the extracellular signal-regulated kinase mediated pathway and inhibits inflammatory cytokines and chemokines in epithelial cells from infertile women with recurrent Chlamydia trachomatis infection. DNA Cell Biol, 2011, 30: 545-554

26 Vignola M J, Kashatus D F, Taylor G A, et al. cPLA2 regulates the expression of type I interferons and intracellular immunity to Chlamydia trachomatis. J Biol Chem, 2010, 285: 21625-21635

27 Darville T, O'Neill J M, Andrews C W Jr, et al. Toll-like receptor-2, but not Toll-like receptor-4, is essential for development of oviduct pathology in chlamydial genital tract infection. J Immunol, 2003, 171: 6187-6197

28 He X, Nair A, Mekasha S, et al. Enhanced virulence of Chlamydia muridarum respiratory infections in the absence of TLR2 activation. PLoS ONE, 2011; 6: e20846

29 O'Connell C M, AbdelRahman Y M, Green E, et al. Toll-like receptor 2 activation by Chlamydia trachomatis is plasmid dependent, and plasmid-responsive chromosomal loci are coordinately regulated in response to glucose limitation by $C$. trachomatis but not by $C$. muridarum. Infect Immun, 2011, 79: 1044-1056

Open Access This article is distributed under the terms of the Creative Commons Attribution License which permits any use, distribution, and reproduction in any medium, provided the original author(s) and source are credited. 